\title{
Unbalanced expression of membrane-bound and soluble OX40 and OX40 ligand in patients with myasthenia gravis
}

Xiaoling Zhou, Qun Xue, et $\mathrm{al}^{1}$

${ }^{1}$ Affiliation not available

September 13, 2021

Xiaoling Zhou ${ }^{1}$, Xiaoyuan Wang ${ }^{1}$, Yanzheng $\mathrm{Gu}^{2}$, Lan $\mathrm{Chen}^{3}$, Yueping Shen ${ }^{4}$, Jingluan Tian ${ }^{1}$, Mingyuan Wang $^{5}$, Shujun Chen ${ }^{1}$, Xiaoyu Duan ${ }^{1}$, Hanqing Gao ${ }^{1}$, Xiaopei Ji ${ }^{1}$, Qi Fang ${ }^{1,2}$, Xueguang Zhang ${ }^{2,3}$, Qun $\mathrm{Xue}^{* 1,2}$

1. Department of Neurology, First Affiliated Hospital of Soochow University, Suzhou, Jiangsu, 215006, China.

2. Institute of Clinical Immunology, Jiangsu Key Laboratory of Clinical Immunology, First Affiliated Hospital of Soochow University, Suzhou, Jiangsu, 215006, China.

3. Department of Neurology, Nantong First People's Hospital, Nantong, Jiangsu, 226000, China.

4. Department of Epidemiology and Health Statistics, Soochow University, Suzhou, Jiangsu, 215006, China.

5. Suzhou Red Cross Central Blood Station, Suzhou, Jiangsu, 215006, China.

*Correspondence Author: Qun Xue (email: qxue_sz@163. com)

Short title: Clinical significance of OX40 and OX40L in myasthenia gravis

\section{Abbreviations}

MG: myasthenia gravis

USMG: untreated stage myasthenia gravis

RSMG: recurrence stage myasthenia gravis

PSMG: paracmasis stage myasthenia gravis

HC: healthy control

PB: peripheral blood

mOX40: membrane-bound OX40

mOX40L: membrane-bound OX40L

sOX40: soluble OX40

sOX40L: soluble OX40L

ELISA: enzyme-linked immunosorbent assay

OMG: ocular myasthenia gravis 
GMG: generalized myasthenia gravis

CT: computed tomography

QMGs: quantitative myasthenia gravis scores

AchR-Ab: acetylcholine receptor antibodies

MHC: major histocompatibility complex

TNFR: tumour necrosis factor receptor

TNF: tumour necrosis factor

ADCs: antigen-presenting cells

DCs: dendritic cells

MS: multiple sclerosis

SLE: systemic lupus erythematosus

RA: rheumatoid arthritis

MGFA: Myasthenia Gravis Foundation of America

ROC: receiver operating characteristic

CI: confidence interval

\section{Summary}

Myasthenia gravis (MG) is a T cell-dependent, antibody-mediated autoimmune disease. The OX40/OX40L pathways play a crucial role in the pathogenesis or development of human autoimmune diseases. This study aimed to investigate the functions, potential mechanisms, and clinical significance of the OX40/OX40L pathways in the pathological process of MG. Ninety patients diagnosed with MG, as well as 39 patients with untreated stage MG (USMG), 22 patients with recurrence stage MG (RSMG), 42 patients with paracmasis stage MG (PSMG), and 36 healthy controls (HC), were enrolled in this study. Peripheral blood (PB) was collected from patients with MG and HCs. The expression of membrane-bound OX40 (mOX40) and OX40L (mOX40L) on immune cells was detected using flow cytometry. The levels of soluble OX40 (sOX40) and OX40L (sOX40L) in plasma were analyzed using ELISA. The expression levels of OX40 on T cells in peripheral blood from the MG group and OX40L on B cells and mononuclear cells were significantly increased compared to those in the $\mathrm{HC}$ group. The levels of sOX40 were significantly decreased in patients with MG compared to those in HCs, while the levels of sOX40L were not different. A subgroup analysis revealed that the expression of OX40 on $\mathrm{CD}^{+} \mathrm{T}$ cells, OX40L on $\mathrm{CD}^{+} 4^{+}$mononuclear cells and sOX40 in the generalized MG (GMG) group was significantly higher than that in the ocular MG (OMG) group. The expression of OX40 on CD4 ${ }^{+} \mathrm{T}$ cells in patients with thymoma or thymic hyperplasia was significantly upregulated compared with participants without thymoma or thymic hyperplasia. Correlation analyses with clinical data showed that OX40 expression on $\mathrm{CD} 4^{+} \mathrm{T}$ cells was positively correlated with the quantitative myasthenia gravis scores (QMGs) and the concentrations of acetylcholine receptor antibodies (AchR-Ab) in the MG group. sOX40 levels were positively correlated with QMGs and disease duration, while sOX40L levels were negatively correlated with the disease duration. Dynamic observations of the expression of these molecules showed significantly increased expression of OX40 on CD4 ${ }^{+} \mathrm{T}$ cells in the peripheral blood from the USMG, RSMG and PSMG groups compared to that in the HC group. Compared with the USMG and PSMG groups, OX40 expression was significantly increased on $\mathrm{CD}^{+} \mathrm{T}$ cells from the RSMG group. OX40L expression on $\mathrm{CD} 19^{+} \mathrm{B}$ cells was significantly increased in the PSMG group compared with the HC group. OX40L was expressed at significantly higher levels on CD14 ${ }^{+}$mononuclear cells from the RSMG group than on cells from the HC group. However, no significant difference in OX40L expression on CD19 ${ }^{+}$B cells and $\mathrm{CD}^{+}{ }^{+}$mononuclear cells was observed among the USMG, RSMG and PSMG groups. The plasma sOX40 
levels in the USMG group were significantly lower than those in the HC group. Compared with the USMG and PSMG groups, the levels of sOX40 in the RSMG group were significantly increased. sOX40L levels in the PSMG group were significantly lower than those in the USMG, RSMG and HC groups. The expression of OX40 on $\mathrm{CD}^{+} \mathrm{T}$ cells was positively correlated with the concentration of AchR-Ab in the RSMG group, while the expression of OX40L on $\mathrm{CD} 19^{+} \mathrm{B}$ cells and CD14 ${ }^{+}$mononuclear cells was negatively correlated with the disease duration. ROC curves showed that the expression levels of CD4 ${ }^{+}$OX40 and sOX40L had moderate predictive value for monitoring MG recurrence. Based on these results, the OX40/OX40L pathways are involved in the immunopathological process of MG and might be valuable therapeutic targets for MG. Abnormal sOX40L levels might promote the positive signal transduction of OX40 on T cells in the later stages of MG, causing excessive activation of $\mathrm{T}$ cells and disease progression. CD4 ${ }^{+} \mathrm{OX} 40$ and sOX40 may be associated with MG disease activity and severity, and $\mathrm{CD}^{+}{ }^{+} \mathrm{OX} 40$ may be related to the recurrence of MG.

Keywords : myasthenia gravis; peripheral blood; mOX40; mOX40L; sOX40; sOX40L

\section{Introduction}

Myasthenia gravis (MG) is an antibody-mediated autoimmune disease of the neuromuscular junction. Its main clinical manifestations are fluctuating weakness and fatigue of the involved skeletal muscles[1]. At present, the predominant therapeutic strategies for MG include cholinesterase inhibitors, immunosuppressive agents, thymectomy, and short-term immunomodulation via intravenous immunoglobulin or plasma exchange, which are selected according to the clinical characteristics and the types of pathogenic antibodies [2]. Based on in-depth studies of the pathogenesis of MG, the use of targeted biological agents such as rituximab[3] improves the prognosis of patients with MG. Nevertheless, some patients with refractory MG still have a poor prognosis after humoral immune intervention[4], suggesting that both humoral and cellular immunity may play a key role in the pathological process of MG.

$\mathrm{T}$ cell activation requires the major histocompatibility complex (MHC)-peptide complex to provide a first signal and a second signal delivered by costimulatory molecules. A lack of costimulatory signals can lead to $\mathrm{T}$ cells that are unable to respond and even programmed cell death[5]. OX40 (also named CD134, TNFRSF4 or ACT35) and its cognate ligand, OX40L (also called CD252, TNFSF4, gp34 or CD134L), which are members of the tumor necrosis factor receptor (TNFR) and the tumor necrosis factor (TNF) superfamily, respectively, play an important role in regulating the immune response. OX40 is a type I transmembrane glycoprotein that is mainly expressed on activated $\mathrm{CD} 4^{+} \mathrm{T}$ cells. OX40L is a type II transmembrane glycoprotein that is predominantly expressed on antigen-presenting cells (ADCs), such as B cells, dendritic cells (DCs) and macrophages[6, 7]. OX40-OX40L interactions promote T cell proliferation, differentiation, memory, and survival, increase effector cytokine secretion, and suppress regulatory $\mathrm{T}$ cell function[8]. The OX40/OX40L pathway plays a significant role in the pathogenesis of human autoimmune diseases, including multiple sclerosis (MS) [9], systemic lupus erythematosus (SLE)[10], rheumatoid arthritis (RA)[11], and type 1 diabetes[12], and the expression of $\mathrm{OX} 40$ on $\mathrm{CD}^{+} \mathrm{T}$ cells correlates with disease severity in patients with SLE[13, 14]. Blockade of OX40-OX40L interactions ameliorates disease in many animal models of autoimmunity[15].

In addition to membrane-bound OX40 (mOX40) and membrane-bound OX40L (mOX40L) expression on peripheral circulating lymphocytes, soluble forms of OX40 (sOX40) and OX40L (sOX40L) have been detected in plasma[8]. Soluble molecules regulate the OX40/OX40L axis by binding to corresponding membranebound molecules and increase the diversity and complexity of the OX40/OX40L pathways. However, the specific functions and pathogenesis of the OX40/OX40L pathways in MG remain unclear, and little research has been conducted on soluble molecules. Investigations of whether OX40/OX40L (including membranebound and/or soluble forms) are abnormally expressed in the peripheral blood of patients with MG and whether the abnormal changes are relevant to the onset, recurrence, remission, and activity and severity of MG are worthwhile. This study detected the expression of mOX40 and mOX40L on the surface of peripheral lymphocytes from patients with MG and healthy controls (HCs) and the plasma levels of sOX40 and sOX40L to explore the functions, potential mechanisms, and clinical implications of OX40/OX40L signaling in the 
occurrence and development of MG.

\section{Materials and methods}

\section{Patients and Controls}

Between July 2019 and April 2021, 90 patients with MG were enrolled from the Department of Neurology of the First Affiliated Hospital of Soochow University. All patients were diagnosed with MG according to typical clinical features, neostigmine tests, repetitive nerve electrical stimulation, fatigue tests and positive acetylcholine receptor antibody (AChR-Ab) results. Simultaneously, 36 healthy controls (HCs) matched to patients with MG for sex, age, and race were enrolled from the hospital's physical examination center. The study subjects were excluded if they had one of the following conditions: other autoimmune diseases, acute infectious diseases, malignant tumor (except thymoma), mental illness, or pregnancy or lactation. The quantitative $\mathrm{MG}$ score (QMG) was used to assess the disease severity, and all patients received standard treatment after admission to the hospital. The clinical characteristics of the study subjects are shown in Table 1.

Patients with MG were classified into different subgroups based on sex, age at onset, Myasthenia Gravis Foundation of America (MGFA) classification, thymic histology, and AchR-Ab results. According to the MGFA classification[16], patients were classified as having ocular MG (OMG) or generalized MG (GMG). All patients underwent enhanced chest computed tomography (CT) examinations and were divided into an abnormal thymus group (with thymoma or thymic hyperplasia) and a normal thymus group (without thymoma or thymic hyperplasia). Due to the low positive proportion of anti-MuSK antibodies in the Chinese population[17], the concentrations of AchR-Ab (positive cut-off value was $0.45 \mathrm{nmol} / \mathrm{L}$ ) were detected in all patients with MG, and patients were divided into the AchR-Ab-positive group and the AchR-Ab-negative group (cut-off value of $0.45 \mathrm{nmol} / \mathrm{L}$ ).

Thirty-nine patients with untreated stage MG (USMG), 22 patients with recurrence stage MG (RSMG), and 42 patients with paracmasis stage MG (PSMG) were recruited from the Department of Neurology of the First Affiliated Hospital of Soochow University between July 2019 and June 2021. USMG was defined as the first onset without treatment, including glucocorticoids, immunosuppressants, plasma-exchange therapy or intravenous immunoglobulins in the past 3 months. RSMG was defined as the exacerbation of the original symptoms or signs or the recurrence of symptoms after improvement. The duration of recurrence exacerbations should be longer than $24 \mathrm{~h}$, and the QMGs should be increased. PSMG was defined as the absence of symptoms or signs of MG, with or without weakness of eyelid closure, and no weakness of any other muscle upon careful examination[18]. The demographic and clinical characteristics of all study populations are summarized in

\section{Supplementary Table 2.}

This study was approved by the Ethics Committee of Soochow University, China. All study participants signed informed consent forms.

\section{Sample processing}

Four milliliters of fasting venous blood were collected into 2 EDTA-anticoagulant tubes from all subjects in the morning. One tube was used for the flow cytometry analysis of lymphocytes. One tube was centrifuged at $3000 \mathrm{rpm}$ for 20 minutes, after which the top-layer plasma sample was collected and frozen at $-80{ }^{\circ} \mathrm{C}$ for future use.

\section{Immunofluorescence labeling and flow cytometry analysis}

Peripheral blood $(50 \mu \mathrm{l})$ was collected from each subject, and the following fluorescently labeled monoclonal antibodies were added: FITC-conjugated anti-human CD19, FITC-conjugated anti-human 14, FITCconjugated anti-human OX40, PE-conjugated anti-human OX40L, and PC5-conjugated anti-human CD4 (all purchased from BioLegend, San Diego, CA, USA), followed by an incubation in the dark at room temperature for 30 minutes. Next, $200 \mu \mathrm{l}$ of red blood cell lysis buffer (Beckman Coulter, Brea, CA, USA) were 
added, and the mixtures were incubated at $37^{\circ} \mathrm{C}$ for 10 minutes. Finally, each specimen was washed with 1 $\mathrm{ml}$ of PBS, centrifuged at $2000 \mathrm{rpm}$ for 5 minutes, resuspended in $500 \mu \mathrm{l}$ of PBS, and detected using a flow cytometer (Beckman Coulter, Brea, CA, USA). FlowJo version 10.4 software was used to analyze the raw flow cytometry data.

\section{ELISAs}

Plasma samples from 78 of 103 patients with MG were selected (no statistically significant differences in sex, age, MGFA classification and thymic histology of patients from whom specimens were selected were observed compared with the general population), and plasma from 30 of 36 healthy volunteers was. Detailed information regarding the samples is presented in Supplementary Table 1. Meanwhile, plasma samples from 37 patients with USMG, 34 patients with RSMG and 30 patients with PSMG were selected from the plasma sample bank of the First Affiliated Hospital of Soochow University from July 2017 to May 2021, and the clinical characteristics of patients with different stages of MG are shown in Supplementary Table 3. In addition, plasma sOX40 and sOX40L expression levels were detected in 13 patients with MG in the recurrence and remission stages. The frozen plasma specimens were thawed at room temperature and centrifuged at $2000 \mathrm{rpm}$ for 5 minutes, and the plasma supernatant was taken for enzyme-linked immunosorbent assay (ELISA). The concentrations of AchR-Ab were detected in plasma samples from all patients with MG using the Human Acetylcholine Receptor Autoantibody ELISA Kit (RSR Biotechnology Co., Ltd., UK). Plasma levels of sOX40 and sOX40L were measured using ELISAs (human sOX40 and sOX40L reagent kits were obtained from Shanghai Kang Lang Biological Technology Co., Ltd., Shanghai, China). The specific experimental procedures used for ELISAs were performed according to the manufacturer's instructions.

\section{Statistical analyses}

SPSS version 26.0 and GraphPad Prism 8.0 software were used for statistical analyses. Quantitative data are reported as the means and standard deviations (normal distribution) or medians and interquartile ranges (nonnormal distribution). The T-test (normal distribution) and Mann-Whitney U test (nonnormal distribution) were used to compare independent samples between 2 groups. Comparisons among multiple groups of samples were analyzed using one-way ANOVA (normal distribution) and the Kruskal-Wallis H test (nonnormal distribution), while the Bonferroni-corrected Mann-Whitney U test was used for intergroup comparisons. Qualitative data are reported as frequencies and percentages. The chi-square test or Fisher's exact test was performed to compare qualitative variables. The nonparametric Spearman correlation analysis was used to analyze the correlations between two continuous variables. A $P$ value $<0.05$ was considered statistically significant. A receiver operating curve (ROC) was constructed to evaluate the efficacy of the expression levels of different molecules in predicting MG recurrence.

\section{Results}

OX40/OX40L expression was increased on lymphocytes in the peripheral blood from patients with MG

Flow cytometry was used to analyze the expression of OX40 and OX40L on the peripheral blood lymphocytes of all subjects. The expression of OX40 on $\mathrm{CD} 4^{+} \mathrm{T}$ cells in the MG group was significantly higher than that in the HC group (Fig. 1A, B ). Compared with the HC group, OX40L expression was significantly increased on $\mathrm{CD} 19^{+} \mathrm{B}$ cells and CD14 ${ }^{+}$monocytes from the MG group (Fig. 1A, C, D ). The levels of mOX40 and mOX40L in the MG group and HC group are presented inSupplementary Table 4 .

OX40 was expressed at significantly higher levels on $\mathrm{CD} 4^{+} \mathrm{T}$ cells from the USMG, RSMG and PSMG groups than on cells from the HC group. Compared with the USMG and PSMG groups, OX40 expression on $\mathrm{CD}^{+} \mathrm{T}$ cells from the RSMG group was significantly increased (Fig. 3A, B ). OX40L expression on $\mathrm{CD}_{1}{ }^{+} \mathrm{B}$ cells was significantly increased in the PSMG group compared with the HC group (Fig. 3A, C ). Higher expression of OX40L was detected on $\mathrm{CD} 14^{+}$monocytes from the RSMG group than on cells from the HC group (Fig. 3A, D ). However, no significant difference in OX40L expression on CD19 ${ }^{+} \mathrm{B}$ cells and $\mathrm{CD}_{14}{ }^{+}$mononuclear cells was observed among the USMG, RSMG and PSMG groups. The levels of mOX40 
and mOX40L in patients with different stages of MG are shown in Supplementary Table 6 .

\section{Serum levels of sOX40, but not of sOX40L, were decreased in patients with MG}

Plasma sOX40 and sOX40L levels were measured using ELISAs to explore the potential roles of soluble costimulatory molecules in patients with MG. Plasma sOX40 levels in the MG group were significantly decreased compared with those in the HC group (Fig. 1E ). Nevertheless, no significant difference in the plasma sOX40L levels was observed between the MG group and HC group (Fig. 1F ). The sOX40 and sOX40L levels in the MG group and HC group are displayed in Supplementary Table 5 .

Significantly lower plasma sOX40 levels were detected in the USMG group than in the HC group. sOX40 expression levels in the RSMG and PSMG groups were not significantly different compared with the HC group. Compared with the USMG and PSMG groups, the levels of sOX40 in the RSMG group were significantly increased. The levels of sOX40 in the PSMG group were significantly higher than those in the USMG group (Fig. 3E ). sOX40L levels in the PSMG group were significantly decreased compared with those in the HC group. However, compared with the HC group, the levels of sOX40L in the RSMG group were not significantly different from those in the USMG and RSMG groups. The expression levels of sOX40L in the PSMG group were significantly lower than those in the USMG and RSMG groups (Fig. 3F ). The levels of sOX40 and sOX40L in patients with different stages of MG are shown inSupplementary Table 7 .

The levels of sOX40 and sOX40L in 13 patients with MG in the recurrence stage were significantly higher than those in patients in the paracmasis stage (Supplementary Table 8, Fig. 4D, F ).

\section{Subgroup analysis}

A subgroup analysis was performed to assess the relationship between the expression levels of costimulatory molecules (mOX40/mOX40L and sOX40/sOX40L) and clinical parameters in patients with MG. Ninety patients with MG were divided into 5 subgroups according to sex, age of onset, MGFA classification, thymic histology, and AchR-Ab results. Specifically, significantly higher OX40 expression was observed on CD4 ${ }^{+}$ $\mathrm{T}$ cells in the GMG group than in the OMG group and in patients with thymoma or thymic hyperplasia than in those without. No significant differences were observed between groups stratified by sex, age of onset or AchR-Ab levels. In terms of the expression of OX40L on CD19+ $\mathrm{B}$ cells in patients with MG, no statistically significant differences were observed between all subgroups. Regarding the expression of OX40L on $\mathrm{CD} 14^{+}$mononuclear cells in patients with MG, the GMG group exhibited significantly higher expression than the OMG group, and significant differences were not observed between groups stratified by sex, age of onset, thymic histology and AchR-Ab levels. Results of the subgroup analysis of mOX40 and mOX40L levels in patients with MG are summarized in Table 2 .

The levels of sOX40 were significantly increased in patients with GMG compared to patients with OMG, and no significant differences were observed between groups stratified by sex, age of onset, thymic histology and AchR-Ab levels. However, in the analysis of the expression levels of sOX40L in patients with MG, no statistically significant differences were detected between all subgroups. Results of the subgroup analysis of sOX40 and sOX40L levels in patients with MG are presented inTable $\mathbf{3}$.

\section{Correlation with clinical data}

We analyzed the correlations between the laboratory parameters and clinical data (including age, QMGs, the concentration of AchR-Ab and the disease duration) in the patients with MG to explore the clinical significance of the expression of mOX40/mOX40L and sOX40/sOX40L. In the MG group, the expression of OX40 on $\mathrm{CD}^{+} \mathrm{T}$ cells and sOX40 levels were positively correlated with the QMGs (Fig. 2A, J ). The expression of $\mathrm{OX} 40$ on $\mathrm{CD}^{+} \mathrm{T}$ cells was positively correlated with the concentration of AchR-Ab (Fig. 2B ). A trend toward a positive correlation was observed between the sOX40 levels and disease duration (Fig. 2L ) and a trend toward a negative correlation was observed between the sOX40L levels and disease duration (Fig. 2O ). The correlation analysis results for the MG group are shown in Table 4 and Fig. 2 .

Furthermore, in the RSMG group, the expression of OX40 on CD4 ${ }^{+} \mathrm{T}$ cells positively correlated with the 
AchR-Ab concentration (Fig. 4A ), while OX40L expression on CD19+ $\mathrm{B}$ cells and $\mathrm{CD} 14^{+}$monocytes was negatively correlated with the disease duration (Supplementary Table 10, Fig. 4B, C ). The expression of membrane-bound and soluble OX40/OX40L showed no significant correlation with clinical data in the USMG and PSMG groups (Supplementary Table 9, Supplementary Table 11 ).

\section{Diagnostic and prognostic value of CD4 ${ }^{+} \mathrm{T}$ cell OX40 and sOX40L expression in patients with MG}

We generated a receiver operating characteristic (ROC) curve using the expression data from patients with RSMG and patients with PSMG to evaluate the diagnostic and prognostic value of OX40 and sOX40L levels in $\mathrm{CD} 4{ }^{+} \mathrm{T}$ cells. The area under the ROC curve for the $\mathrm{CD} 4^{+} \mathrm{T}$ cell OX40 index was $0.805[95 \%$ confidence interval (CI): $0.688-0.922, P=0.000]$, the sensitivity was $59 \%$, and the specificity was $93 \%$ (Fig. $4 \mathbf{E}$ ). The area under the ROC curve for the sOX40L index was 0.784 (95\% CI: $0.671-0.898, P=0.000)$, the sensitivity was $68 \%$, and the specificity was $90 \%$ (Fig. 4G ). The results indicated that OX40 and sOX40L levels in $\mathrm{CD} 4^{+} \mathrm{T}$ cells have moderate predictive value in monitoring recurrence in patients with MG.

\section{Discussion}

OX40, a member of the TNFR superfamily, is mainly expressed on activated CD4 ${ }^{+} \mathrm{T}$ cells. Its cognate ligand OX40L, a member of the TNF superfamily, is predominantly expressed on activated ADCs and on some endothelial cells, mast $\mathrm{T}$ cells and activated T cells. Accumulating evidence has shown that the OX40OX40L pathway plays a crucial role in the pathogenesis of multiple autoimmune diseases[19-22]. Furthermore, a correlation between the expression of OX40 on CD4 ${ }^{+} \mathrm{T}$ cells and disease severity has been observed in individuals with autoimmune diseases such as SLE[13, 23]. The OX40-OX40L interaction contributes to promoting the activation, proliferation and long-term survival of effector $\mathrm{T}$ cells, preserving cellular memory, facilitating the production of effector cytokines, and suppressing regulatory function by activating the PI3K$\mathrm{PKB} / \mathrm{NF}-\boldsymbol{x B} / \mathrm{NFAT}$ pathways[7]. Here, we detected the expression of membrane-bound and soluble OX40 and OX40L and explored the functions, and potential mechanisms, as well as the clinical significance of OX40/OX40L signaling in the occurrence and development of MG.

In this study, we found that the expression of the positive costimulatory molecule OX40 on CD4 ${ }^{+} \mathrm{T}$ cells from patients with MG was significantly higher than that on cells from the HC group, and OX40 expression on $\mathrm{CD}^{+} \mathrm{T}$ cells was positively correlated with the concentration of AchR-Ab, consistent with previous research results[24]. In contrast previous studies or new findings, CD4 ${ }^{+}$OX40 expression on $\mathrm{CD} 4^{+} \mathrm{T}$ cells from patients with MG was not related to the age at onset in our study[24], which may be associated with the sample size and disease activity. In addition, our study showed that OX40L expression on CD19+ $\mathrm{B}$ cells and $\mathrm{CD}_{14}{ }^{+}$mononuclear cells in the $\mathrm{MG}$ group was significantly upregulated compared with that in the HC group. The subgroup analysis revealed significantly higher expression of OX40 on $\mathrm{CD} 4^{+} \mathrm{T}$ cells from the GMG group than on cells from the the OMG group and patients with thymoma or thymic hyperplasia than in those without thymoma or thymic hyperplasia, and OX40L expression was significantly increased on $\mathrm{CD} 14^{+}$mononuclear cells from the GMG group compared with that in the OMG group. The correlation analysis showed that OX40 expression on CD4 ${ }^{+} \mathrm{T}$ cells was positively correlated with QMGs. Based on these results, the expression of OX40 on $\mathrm{CD}^{+}{ }^{+} \mathrm{T}$ cells may be related to the severity of MG, and the OX40/OX40L signal participates in the immunopathological process of MG. To the best of our knowledge, the present study is the first to discover increased expression of OX40/OX40L on the peripheral blood lymphocytes of patients with MG. Therefore, we speculate that the OX40-OX40L interactions may contribute to the activation, proliferation and long-term survival of effector T cells, promote B cell differentiation to secrete autoantibodies and inhibit cell apoptosis in patients with MG, which may generate immune disorders and immune injury.

Because MG is an intricate, heterogeneous disease, the expression of these molecules may dynamically change across the disease course. In the present study, significantly higher OX40 expression was detected on CD4 ${ }^{+}$ T cells from the USMG, RSMG and PSMG groups than on cells from the HC group. OX40L expression on $\mathrm{CD} 19^{+} \mathrm{B}$ cells was significantly increased in the PSMG group compared with the HC group. OX40L was 
expressed at higher levels on $\mathrm{CD} 14^{+}$mononuclear cells from the RSMG group than on cells from the HC group. These results indicated that the OX40/OX40L pathways in MG mainly play regulatory roles in the later stage of the disease. Furthermore, compared with the USMG and PSMG groups, the expression of OX40 on $\mathrm{CD} 4^{+} \mathrm{T}$ cells from the RSMG group was significantly increased. The results of the correlation analysis indicate that OX40 expression on $\mathrm{CD} 4^{+} \mathrm{T}$ cells was positively correlated with the AchR-Ab concentration, while OX40L expression on $\mathrm{CD} 19^{+} \mathrm{B}$ cells and $\mathrm{CD} 14^{+}$mononuclear cells was negatively correlated with the disease duration in the RSMG group. These results suggested that the expression of OX40 on $\mathrm{CD}^{+} \mathrm{T}$ cells may be closely related to the recurrence of MG. These phenomena may be related to the mechanism of OX40/OX40L signaling in T cells. On the one hand, from the perspective of T cell survival, Song, SalekArdakani $[25]$ et al. found that OX40-deficient T cells normally differentiated and proliferated into effector T cells 2-3 days after the activation of TCR signaling. Nevertheless, the survival rate was significantly decreased after 12-13 days of activation, suggesting that the OX40/OX40L pathways might not affect the early stage of $\mathrm{T}$ cell proliferation but promote the activation of $\mathrm{T}$ cells and prolong their survival in the later stage. On the other hand, from the perspective of T cell function, OX40-OX40L interactions promote the generation of memory $\mathrm{T}$ cells and maintain their survival[26-29], and the long-term survival of memory $\mathrm{CD}^{+} \mathrm{T}$ cells after antigen restimulation promotes their rapid differentiation into effector $\mathrm{T}$ cells, which may be one of the causes of disease recurrence [30].

In addition to the membrane-bound forms, costimulatory molecules also exist in soluble forms. Soluble costimulatory molecules are generated through the proteolytic cleavage[31] or mRNA splicing[32] of membranebound molecules. sOX40 and sOX40L may be cleaved from membrane molecules, but the specific mechanism remains unclear[12, 33]. We detected the plasma levels of sOX40 and sOX40L in patients with MG for the first time to explore the roles of the soluble molecules. The plasma sOX40 levels in the MG group, especially in the USMG group, were significantly decreased compared with those in the HC group, consistent with the findings of unbalanced expression of the membrane and soluble form of OX40 in individuals with type 1 diabetes[12]. Moreover, plasma sOX40 levels were positively correlated with QMGs and disease duration in the MG group. Therefore, sOX40 may be associated with disease severity in patients with MG. Studies have confirmed that sOX40 binds to mOX40L on ADCs to interfere with the positive signals transduced by the OX40/OX40L pathway and suppress T cell activation [34-36]. Therefore, we speculated that activated $\mathrm{T}$ cells may be inhibited by sOX40-induced blockade of OX40/OX40L, and then inactivated T cells would generate less sOX40 in the early stage of MG. In the dynamic process of disease progression, similar to mOX40, the levels of sOX40 in the RSMG group were significantly higher than those in the USMG and PSMG groups, and the level of sOX40 in 13 patients with recurrent MG was significantly higher than that in patients in remission. Based on the findings, we hypothesized that the increase in sOX40 levels in the RSMG group may be due to increased shedding from mOX40.

No significant difference in the plasma sOX40 levels was observed between the MG group and HC group. The dynamic observation of the molecules showed that sOX40L levels in the PSMG group were significantly decreased compared with those in the HC, USMG and RSMG groups. Moreover, the levels of sOX40L in 13 patients with MG in the remission stage were significantly lower than those in the recurrence stage. The correlation analysis showed that sOX40L levels were negatively correlated with the disease duration. Experiments have shown that sOX40L promotes late proliferation and activation states of $\mathrm{T}$ cells but does not influence early proliferation and activation[37, 38]. Researchers have speculated that the binding of sOX40L in the peripheral blood to OX40 on activated $\mathrm{T}$ cells would allow $\mathrm{T}$ cells to receive continuous persistent and positive stimulation signals[39, 40], leading to relative immune system hyperactivity in the later stages of MG. Excessive consumption of sOX40L may result in decreased sOX40L levels in later disease stages.

In our study, the concentration of sOX40L was dozens of times higher than that of sOX40. We hypothesized that sOX40L may be a functional molecule and may enhance the positive signals transduced by the OX40/OX40L pathway in the later stage of MG, which may be an important mechanism for reinitiating an immune response, but this hypothesis requires further exploration in the future. ROC curves showed the moderate predictive value of the expression levels of OX40 and sOX40L in CD4 ${ }^{+} \mathrm{T}$ cells for monitoring $\mathrm{MG}$ 
recurrence. However, the sensitivity was low, and further large-scale cohort studies should be conducted in the future.

In summary, this study suggested that abnormal activation of the OX40/OX40L pathway participates in the immunopathological process of MG. sOX40L may regulate the positive signals transduced by the OX40/OX40L pathway in the later stage of the disease, leading to the activation and proliferation of effector $\mathrm{T}$ cells and the subsequent progression of MG. Membrane-bound and soluble OX40 expression levels are correlated with MG disease activity and severity, and OX40 expression on CD4 ${ }^{+} \mathrm{T}$ cells may be related to the recurrence of MG. Further studies targeting the specific mechanisms underlying the involvement of the OX40/OX40L pathway in MG-related immune disorders will provide new targets and directions for the treatment of MG.

\section{Acknwledgements}

This study was supported by the Priority Academic Program Development of Jiangsu Higher Education Institutions of China, the Key R\&D Program of Jiangsu Province (BE2019666)

\section{Author contributions}

YG and QX designed the study. XZ, XW, XD, HG and XJ collected the samples of patients with myasthenia gravis. XZ, SC, JT and LC performed experiments. YG and XZ instructed and supervised laboratory experiments. YS, JT, XJ and HG analyzed the data. QF and MW provided funding and administrative support. XZ wrote the manuscript. QX revised the manuscript. All authors reviewed and approved the revised manuscript.

\section{Conflicting interest}

The authors have no conflicts of interest to declare.

\section{Data availability statement}

The authorship will provide all raw data supporting the conclusions of this manuscript without any reservation.

\section{References}

1. Gilhus NE. Myasthenia Gravis. N Engl J Med 2016;375 :2570-81.

2. Narayanaswami P, Sanders DB, Wolfe G, Benatar M, Cea G, Evoli A, Gilhus NE, Illa I, Kuntz NL, Massey J, Melms A, Murai H, Nicolle M, Palace J, Richman D, Verschuuren J. International Consensus Guidance for Management of Myasthenia Gravis: 2020 Update. Neurology 2021;96 :114-22.

3. Di Stefano V, Lupica A, Rispoli MG, Di Muzio A, Brighina F, Rodolico C. Rituximab in AChR subtype of myasthenia gravis: systematic review. J Neurol Neurosurg Psychiatry 2020; 91 :392-5.

4. Brauner S, Eriksson-Dufva A, Hietala MA, Frisell T, Press R, Piehl F. Comparison Between Rituximab Treatment for New-Onset Generalized Myasthenia Gravis and Refractory Generalized Myasthenia Gravis.JAMA Neurol 2020; 77 :974-81.

5. Leitner J, Grabmeier-Pfistershammer K, Steinberger P. Receptors and ligands implicated in human T cell costimulatory processes.Immunol Lett 2010; $128: 89-97$.

6. Fu Y, Lin Q, Zhang Z, Zhang L. Therapeutic strategies for the costimulatory molecule OX40 in T-cellmediated immunity. Acta Pharm Sin B 2020; $10: 414-33$.

7. Edner NM, Carlesso G, Rush JS, Walker LSK. Targeting co-stimulatory molecules in autoimmune disease. Nat Rev Drug Discov 2020;19:860-83.

8. Webb GJ, Hirschfield GM, Lane PJ. OX40, OX40L and Autoimmunity: a Comprehensive Review. Clin Rev Allergy Immunol 2016;50 :312-32. 
9. Carboni S, Aboul-Enein F, Waltzinger C, Killeen N, Lassmann H, Pena-Rossi C. CD134 plays a crucial role in the pathogenesis of EAE and is upregulated in the CNS of patients with multiple sclerosis. J Neuroimmunol 2003; 145 :1-11.

10. Jacquemin C, Augusto JF, Scherlinger M, Gensous N, Forcade E, Douchet I, Levionnois E, Richez C, Lazaro E, Duffau P, Truchetet ME, Seneschal J, Couzi L, Pellegrin JL, Viallard JF, Schaeverbeke T, Pascual V, Contin-Bordes C, Blanco P. OX40L/OX40 axis impairs follicular and natural Treg function in human SLE. JCI Insight 2018; 3 .

11. Croft M, Siegel RM. Beyond TNF: TNF superfamily cytokines as targets for the treatment of rheumatic diseases. Nat Rev Rheumatol 2017;13 :217-33.

12. An J, Ding S, Li S, Sun L, Chang X, Huang Z, Zhou B, Fang C, Liu C, Zhang X. Enhancement of the Soluble Form of OX40 and OX40L Costimulatory Molecules but Reduction of the Membrane Form in Type 1 Diabetes (T1D). J Immunol Res 2019; 2019 :1780567.

13. Farres MN, Al-Zifzaf DS, Aly AA, Abd Raboh NM. OX40/OX40L in systemic lupus erythematosus: association with disease activity and lupus nephritis. Ann Saudi Med 2011; 31 :29-34.

14. Kshirsagar S, Binder E, Riedl M, Wechselberger G, Steichen E, Edelbauer M. Enhanced activity of Akt in Teff cells from children with lupus nephritis is associated with reduced induction of tumor necrosis factor receptor-associated factor 6 and increased OX40 expression. Arthritis Rheum 2013; 65 :2996-3006.

15. Fu N, Xie F, Sun Z, Wang Q. The OX40/OX40L Axis Regulates T Follicular Helper Cell Differentiation: Implications for Autoimmune Diseases. Front Immunol 2021; 12 :670637.

16. Jaretzki A, 3rd, Barohn RJ, Ernstoff RM, Kaminski HJ, Keesey JC, Penn AS, Sanders DB. Myasthenia gravis: recommendations for clinical research standards. Task Force of the Medical Scientific Advisory Board of the Myasthenia Gravis Foundation of America. Neurology 2000;55 :16-23.

17. Evoli A, Padua L. Diagnosis and therapy of myasthenia gravis with antibodies to muscle-specific kinase. Autoimmun Rev 2013;12:931-5.

18. Sanders DB, Wolfe GI, Benatar M, Evoli A, Gilhus NE, Illa I, Kuntz N, Massey JM, Melms A, Murai H, Nicolle M, Palace J, Richman DP, Verschuuren J, Narayanaswami P. International consensus guidance for management of myasthenia gravis: Executive summary. Neurology 2016; 87 :419-25.

19. Cui D, Lv Y, Yuan X, Ruan G, Zhang Y, Yan C, Xu D, Lv M, Mao Y, Cao J, Jin J, Xie J. Increased Expressions of OX40 and OX40 Ligand in Patients with Primary Immune Thrombocytopenia. J Immunol Res 2019; 2019 :6804806.

20. Jacquemin C, Schmitt N, Contin-Bordes C, Liu Y, Narayanan P, Seneschal J, Maurouard T, Dougall D, Davizon ES, Dumortier H, Douchet I, Raffray L, Richez C, Lazaro E, Duffau P, Truchetet ME, Khoryati L, Mercie P, Couzi L, Merville P, Schaeverbeke T, Viallard JF, Pellegrin JL, Moreau JF, Muller S, Zurawski S, Coffman RL, Pascual V, Ueno H, Blanco P. OX40 Ligand Contributes to Human Lupus Pathogenesis by Promoting T Follicular Helper Response. Immunity 2015;42 :1159-70.

21. Wang Q, Shi BM, Xie F, Fu ZY, Chen YJ, An JN, Ma Y, Liu CP, Zhang XK, Zhang XG. Enhancement of CD4(+) T cell response and survival via coexpressed OX40/OX40L in Graves' disease. Mol Cell Endocrinol $2016 ; 430: 115-24$.

22. Artinger K, Kirsch AH, Mooslechner AA, Cooper DJ, Aringer I, Schuller M, Schabhuttl C, Klotzer KA, Schweighofer K, Eller P, Yagita H, Illert AL, Rosenkranz AR, Lane PJ, Eller K. Blockade of tumor necrosis factor superfamily members CD30 and OX40 abrogates disease activity in murine immune-mediated glomerulonephritis. Kidney Int 2021;100:336-48.

23. Patschan S, Dolff S, Kribben A, Durig J, Patschan D, Wilde B, Specker C, Philipp T, Witzke O. CD134 expression on $\mathrm{CD} 4+\mathrm{T}$ cells is associated with nephritis and disease activity in patients with systemic lupus 
erythematosus. Clin Exp Immunol 2006; 145 :235-42.

24. Xiaoyan Z, Pirskanen R, Malmstrom V, Lefvert AK. Expression of OX40 (CD134) on CD4+ T-cells from patients with myasthenia gravis.Clin Exp Immunol 2006; 143 :110-6.

25. Song J, Salek-Ardakani S, Rogers PR, Cheng M, Van Parijs L, Croft M. The costimulation-regulated duration of PKB activation controls T cell longevity. Nat Immunol 2004; 5 :150-8.

26. Prell RA, Evans DE, Thalhofer C, Shi T, Funatake C, Weinberg AD. OX40-mediated memory T cell generation is TNF receptor-associated factor 2 dependent. J Immunol 2003; 171 :5997-6005.

27. Vu MD, Clarkson MR, Yagita H, Turka LA, Sayegh MH, Li XC. Critical, but conditional, role of OX40 in memory $\mathrm{T}$ cell-mediated rejection. J Immunol 2006; 176 :1394-401.

28. Soroosh P, Ine S, Sugamura K, Ishii N. Differential requirements for OX40 signals on generation of effector and central memory CD4+ T cells. J Immunol 2007; 179 :5014-23.

29. Gramaglia I, Jember A, Pippig SD, Weinberg AD, Killeen N, Croft M. The OX40 costimulatory receptor determines the development of CD4 memory by regulating primary clonal expansion. J Immunol 2000;165 $: 3043-50$.

30. Croft M, So T, Duan W, Soroosh P. The significance of OX40 and OX40L to T-cell biology and immune disease. Immunol Rev $2009 ; 229$ :173-91.

31. Zhang G, Hou J, Shi J, Yu G, Lu B, Zhang X. Soluble CD276 (B7-H3) is released from monocytes, dendritic cells and activated T cells and is detectable in normal human serum. Immunology 2008;123 :538-46.

32. $\mathrm{Hu} \mathrm{X}, \mathrm{Wu}$ J, An J, Hu Y, Shen Y, Liu C, Zhang X. Development of a novel monoclonal antibody to human inducible co-stimulator ligand (ICOSL): Biological characteristics and application for enzyme-linked immunosorbent assay. Int Immunopharmacol 2016; 36 :151-7.

33. Tanaka Y, Takahashi Y, Tanaka R, Miyagi T, Saito M, Fukushima T. Association of high levels of plasma OX40 with acute adult T-cell leukemia. Int J Hematol 2019; 109 :319-27.

34. Sawada R, Arai Y, Sagawa Y, Nagata Y, Nishimura T, Noguchi M, Amano K, Arihiro S, Saruta M, Homma S. High blood levels of soluble OX40 (CD134), an immune costimulatory molecule, indicate reduced survival in patients with advanced colorectal cancer. Oncol Rep 2019;42 :2057-64.

35. Weinberg AD, Wegmann KW, Funatake C, Whitham RH. Blocking OX-40/OX-40 ligand interaction in vitro and in vivo leads to decreased $\mathrm{T}$ cell function and amelioration of experimental allergic encephalomyelitis. J Immunol 1999; $162: 1818-26$.

36. Higgins LM, McDonald SA, Whittle N, Crockett N, Shields JG, MacDonald TT. Regulation of T cell activation in vitro and in vivo by targeting the OX40-OX40 ligand interaction: amelioration of ongoing inflammatory bowel disease with an OX40-IgG fusion protein, but not with an OX40 ligand-IgG fusion protein. J Immunol 1999;162:486-93.

37. Soroosh P, Ine S, Sugamura K, Ishii N. OX40-OX40 ligand interaction through T cell-T cell contact contributes to CD4 T cell longevity. J Immunol 2006; 176 :5975-87.

38. Rogers PR, Song J, Gramaglia I, Killeen N, Croft M. OX40 promotes Bcl-xL and Bcl-2 expression and is essential for long-term survival of CD4 T cells. Immunity 2001; $15: 445-55$.

39. Sadun RE, Hsu WE, Zhang N, Nien YC, Bergfeld SA, Sabzevari H, Lutsiak ME, Khawli L, Hu P, Epstein AL. Fc-mOX40L fusion protein produces complete remission and enhanced survival in 2 murine tumor models. J Immunother 2008; 31 :235-45.

40. Qin W, Hongya W, Yongjing C, Fang X, Yue M, Xuekun Z, Xiaozhong L, Xueguang Z. Increased OX40 and soluble OX40 ligands in children with Henoch-Schonlein purpura: association with renal involvement.Pediatr Allergy Immunol 2011; 22 :54-9. 


\section{Hosted file}

Figures.docx available at https://authorea.com/users/433652/articles/537053-unbalancedexpression-of-membrane-bound-and-soluble-ox40-and-ox40-ligand-in-patients-withmyasthenia-gravis

\section{Hosted file}

Tables.docx available at https://authorea.com/users/433652/articles/537053-unbalancedexpression-of-membrane-bound-and-soluble-ox40-and-ox40-ligand-in-patients-with-

myasthenia-gravis 